\title{
Complex Zeros of Two Incomplete Riemann Zeta Functions
}

\author{
By K. S. Kölbig
}

\begin{abstract}
The computation of the complex zeros of an incomplete Riemann zeta function defined in an earlier paper is extended and new zero trajectories are given. A second incomplete Riemann zeta function is defined and its zero trajectories are investigated numerically as functions of the upper limit $\lambda$ of the definition integral. It becomes apparent that there exist three different classes of zero trajectories for this function, distinguished by their behaviour for $\lambda \rightarrow \infty$.
\end{abstract}

1. Introduction. Let $s=\sigma+i t$ be a complex variable. In a previous paper [1], some results concerning the complex zeros of an incomplete Riemann zeta function

$$
A(s, \lambda)=\frac{1}{\Gamma(s)} \int_{0}^{\lambda} \frac{x^{s-1}}{e^{x}-1} d x
$$

and of the incomplete gamma function

$$
P(s, \lambda)=\frac{1}{\Gamma(s)} \int_{0}^{\lambda} x^{s-1} e^{-x} d x
$$

were presented, $\lambda>0$ being a real parameter. These results were obtained by a systematic numerical investigation. It became apparent that not all, but only some, of the zero trajectories $\bar{s}(\lambda)$, defined in the s-plane by $A(\bar{s}(\lambda), \lambda)=0$, reach a zero of the Riemann zeta function $\zeta(s)$ on the line $\sigma=\frac{1}{2}$ as $\lambda \rightarrow \infty$. The remaining curves $\tilde{s}(\lambda)$ approach the zero trajectories $\tilde{s}(\lambda)$ of the incomplete gamma function $P(s, \lambda)$, which are defined by $P(\tilde{s}(\lambda), \lambda)=0$.

It is the aim of this paper to present further solutions $\bar{s}(\lambda)$ which again have been obtained by numerical calculation. Because of the fact that there exists the relation

$$
\zeta(s)=\frac{1}{\Gamma(s)} \int_{0}^{\infty} \frac{x^{s-1}}{e^{r}-1} d x=\frac{1}{\left(1-2^{i}-\bar{s}\right) \mathrm{I}(s)} \int_{0}^{\infty} \frac{x^{s-1}}{e^{x}+1} d x,
$$

we introduce, in addition, a second incomplete Riemann zeta function

$$
B(s, \lambda)=\frac{1}{\Gamma(s)} \int_{0}^{\lambda} \frac{x^{s-1}}{e^{x}+1} d x \quad(\sigma>0),
$$

where

$$
\lim _{\lambda \rightarrow \infty} B(s, \lambda)=\left(1-2^{1-s}\right) \zeta(s)=\zeta^{*}(s)
$$

Received July 6, 1971.

AMS 1969 subject classifications. Primary 3345, 6525; Secondary 3315.

Key words and phrases. Incomplete Riemann zeta function, incomplete gamma function, Debye function, complex zeros.

Copyright $\odot$ 1972, American Mathematical Society 
Some of its zero trajectories $\hat{s}(\lambda)$, defined by $B(\hat{s}(\lambda), \lambda)=0$, are also computed.

2. Relations to Other Functions. The incomplete Riemann zeta functions $A(s, \lambda)$ and $B(s, \lambda)$, as functions of a complex variable $s$, do not seem to have arisen so far in applications. However, these functions, the corresponding complete functions $\zeta(s)$ and $\zeta^{*}(s)$, as well as certain of their possible generalizations, play, for other types of variables, under different names, and different notations, an important role in several fields of physics and chemistry, e.g., in thermodynamics and in conductivity theory. They are often called Debye functions, Bose-Einstein functions, Fermi-Dirac functions, etc. In his handbook of special functions, Luke [2] defines the Debye functions by

$$
A^{*}(z, m)=m z^{-m} \int_{0}^{z} \frac{x^{m}}{e^{x}-1} d x,{ }^{*}
$$

for complex $z$ with $\operatorname{Re} z>0$ and positive integral $m$. He also gives rational Padé approximations to these functions. By comparison with (1), we find

$$
A(s, \lambda)=\frac{\lambda^{s-1}}{(s-1) \Gamma(s)} A^{*}(\lambda, s-1) .
$$

Recently, $\mathrm{Ng}$ et al. [3] have defined an incomplete Bose-Einstein function

$$
B_{p}(\eta, u)=\frac{1}{\Gamma(p+1)} \int_{0}^{u} \frac{x^{p}}{e^{x-\eta}-1} d x,
$$

corresponding to a complete function $B_{p}(\eta)$ when $u \rightarrow \infty$. They do not investigate $B_{\nu}(\eta, u)$ further. In another paper, $\mathrm{Ng}$ and Devine [4] present a method for the computation of the Debye functions

$$
\bar{D}_{p}(x)=\frac{1}{p ! p} x^{p} A^{*}(x, p)
$$

for integral $p$ and real $x$. From (1), (8) and (9), we have

$$
A(s, \lambda)=B_{s-1}(0, \lambda)=\bar{D}_{s-1}(\lambda) .
$$

One can also define an incomplete Fermi-Dirac function

$$
F_{p}(\eta, u)=\frac{1}{\Gamma(p+1)} \int_{0}^{u} \frac{x^{p}}{e^{x-\eta}+1} d x,
$$

though this is not done by $\mathrm{Ng}$ et al., who discuss only the complete function $F_{\nu}(\eta)$. If we compare it with $B(s, \lambda)$, we find that

$$
B(s, \lambda)=F_{s-1}(0, \lambda) .
$$

The complete Bose-Einstein functions and the complete Fermi-Dirac functions have been investigated by several authors, e.g., by Dingle [5], [6]. Cody and Thacher [7] have developed rational Chebyshev approximations for $F_{p}(\eta)$ in the particularly important cases $p=-\frac{1}{2}, \frac{1}{2}, \frac{3}{2}$, and real $\eta$. $\mathrm{Ng}$ et al. have given Chebyshev polynomial fusion.

* The asterisk in $A^{*}$ is not in the original notation. We introduce it here in order to avoid con- 
expansions of $B_{p}(\eta)$ for $p=0(1) 10$ and real $\eta$. Finally, we mention the fact that Nielsen's generalized polylogarithms

$$
S_{n, p}(x)=\frac{(-1)^{n+p-1}}{(n-1) ! p !} \int_{0}^{1} \frac{\log ^{n-1} u \log ^{p}(1-x u)}{u} d u,
$$

which are of importance in the theory of Feynman integrals in quantum electrodynamics, and which have recently been discussed by Kölbig et al. [8], for positive integers $n$ and $p$, are connected to the Debye functions and, therefore, to $A(s, \lambda)$. From Eq. (13), with the substitution $u^{\prime}=-\log \left[1-\left(1-e^{-\lambda}\right) u\right]$, we find the relation

$$
A(s, \lambda)=S_{1,0-1}\left(1-e^{-\lambda}\right) \text {. }
$$

It should, however, be noted that, in all these cases, $s$ is considered by the authors to be a real number or even a positive integer. ${ }^{* *}$

3. Other Formulae for $A(s, \lambda)$ and $B(s, \lambda)$. Formulae for $P(s, \lambda)$ and $A(s, \lambda)$ which allow analytic continuation of these functions into regions of the s-plane other than those covered by the definition integrals (1) and (2) are given in [1]. From the power series expansion [9]

$$
\frac{1}{e^{x}+1}=\frac{1}{2} \sum_{n=0}^{\infty} \frac{E_{n}(0)}{n !} x^{n}=\sum_{n=0}^{\infty} \frac{1-2^{n+1}}{(n+1) !} B_{n+1} x^{n},
$$

which converges for $|x|<\pi$, where $E_{n}(\xi)$ is the $n$th Euler polynomial and $B_{n}$ are the Bernoulli numbers, we find for $B(s, \lambda)$, in the same way as for $A(s, \lambda)$ [1], the expression

$$
B(s, \lambda)=\frac{1}{\Gamma(s)}\left\{\frac{1}{2 s}+\sum_{n=1}^{\infty} \frac{1-2^{2 n}}{(2 n) !} \frac{B_{2 n}}{s+2 n-1}+\int_{1}^{\lambda} \frac{x^{-1}}{e^{x}+1} d x\right\}
$$

By analytic continuation, this formula defines $B(s, \lambda)$ in the half plane $\sigma \leqq 0$, except at the integer points $0,-1,-2, \cdots$. By an appropriate limiting procedure, we obtain

$$
B(-k, \lambda)=\left(1-2^{k+1}\right) \zeta(-k)=(-1)^{k}\left(1-2^{k+1}\right) B_{k+1} /(k+1)
$$

for all $\lambda$ and $k=0,1,2, \cdots$. In particular, we have

$$
\begin{array}{ll}
B(0, \lambda)=\frac{1}{2}, & \\
B(-2 k, \lambda)=0 & (k>0), \\
B(-2 k+1, \lambda)=\left(2^{2 k}-1\right) B_{2 k} / 2 k & (k>0) .
\end{array}
$$

For $s=1$, one finds

$$
B(1, \lambda)=\int_{0}^{\lambda} \frac{d x}{e^{x}+1}=\log 2+\lambda-\log \left(e^{\lambda}+1\right)
$$

and

$$
\lim _{\lambda \rightarrow \infty} B(1, \lambda)=\lim _{\iota \rightarrow 1}\left(1-2^{1-\imath}\right) \zeta(s)=\log 2 .
$$

** Of course, it is also possible to introduce the corresponding complementary functions, where the integral is taken from a finite value $\lambda$ to $\infty$ instead of from 0 to $\lambda$. This is done by several authors. We do not present further details here. 
From the expansion

$$
\frac{1}{e^{x}+1}=-\sum_{n=1}^{\infty}(-1)^{n} e^{-n x} \quad(x>0),
$$

we obtain from (4), by using (2) and the substitution $x^{\prime}=n x$, the following expression valid for $\sigma>0$ :

$$
B(s, \lambda)=-\sum_{n=1}^{\infty}(-1)^{n} n^{-\cdot} P(s, n \lambda) .
$$

For $s=\frac{1}{2}$, we can use a known relation between the incomplete gamma function and the error function [9] to obtain

$$
B\left(\frac{1}{2}, \lambda\right)=\sum_{n=1}^{\infty} \frac{(-1)^{n+1}}{\sqrt{ } n} \operatorname{erf}(n \lambda)^{1 / 2} .
$$

The formulae for $A(s, \lambda)$ and $P(s, \lambda)$ which correspond to (16), together with the well-known [9] partial fraction expansion of $P(s, \lambda)$, have been used in [1] for the numerical calculation of these functions. However, for increasing $t$ and $\lambda$, the numerical evaluation of the integrals becomes more and more time-consuming. We present, therefore, another formula for $A(s, \lambda)$, which is essentially due to Putschbach [10], who developed this formula in a manuscript mentioned in [1]. Further, we shall give the corresponding formula for $B(s, \lambda)$.

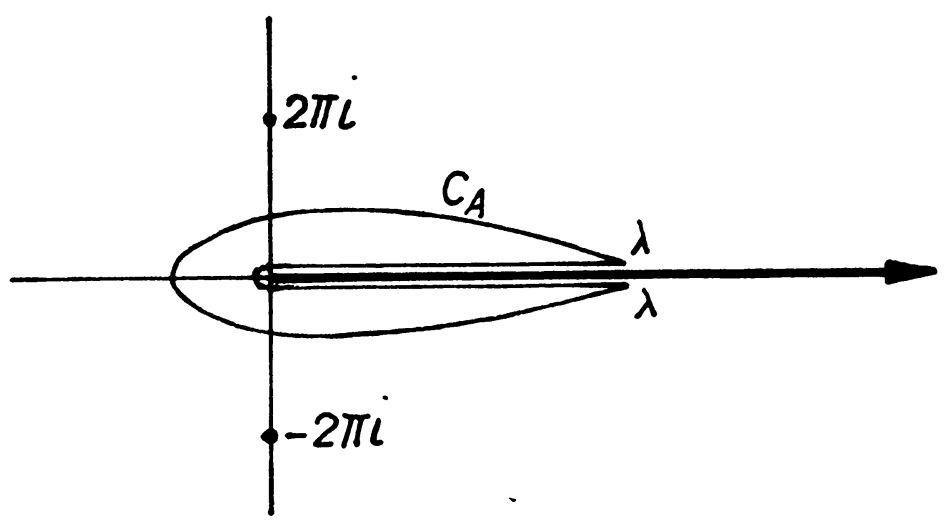

FIGURe 1

We consider a $z$-plane which is cut along the real axis from 0 to $+\infty$ (Fig. 1). In this plane, we construct a contour $C_{A}$ which starts at $\lambda>0$ on the upper boundary of the cut, encircles the origin on the left and ends in $\lambda>0$ on the lower boundary, in such a way that the points $z=2 \pi i$ and $z=-2 \pi i$ remain above and below $C_{A}$, respectively. We then integrate the function

$$
f(z)=(-z)^{s-1} /\left(e^{z}-1\right),
$$

where $(-z)^{s-1}=e^{(0-1) \log (-z)}$ and where the logarithm is taken as real on the negative real axis, along the contour $C_{A}$. By shrinking $C_{A}$ into the boundary of the cut along the real axis, inserting appropriate boundary values along the cut, and using a well- 
known property of the gamma function and the definition (1), we finally obtain

$$
A(s, \lambda)=-\frac{\Gamma(1-s)}{2 \pi i} \int_{C_{\Lambda}} \frac{(-z)^{s-1}}{e^{z}-1} d z
$$

This formula corresponds to the so-called Hankel integral for $\zeta(s)$ and now defines $A(s, \lambda)$ for all $s \neq 1$.

In order to evaluate the contour integral in (25), we deform $C_{A}$ into a circle $K_{\lambda}$ of radius $\lambda$ as shown in Fig. 2, excluding the values $\lambda=2 n \pi(n=1,2,3, \cdots)$.

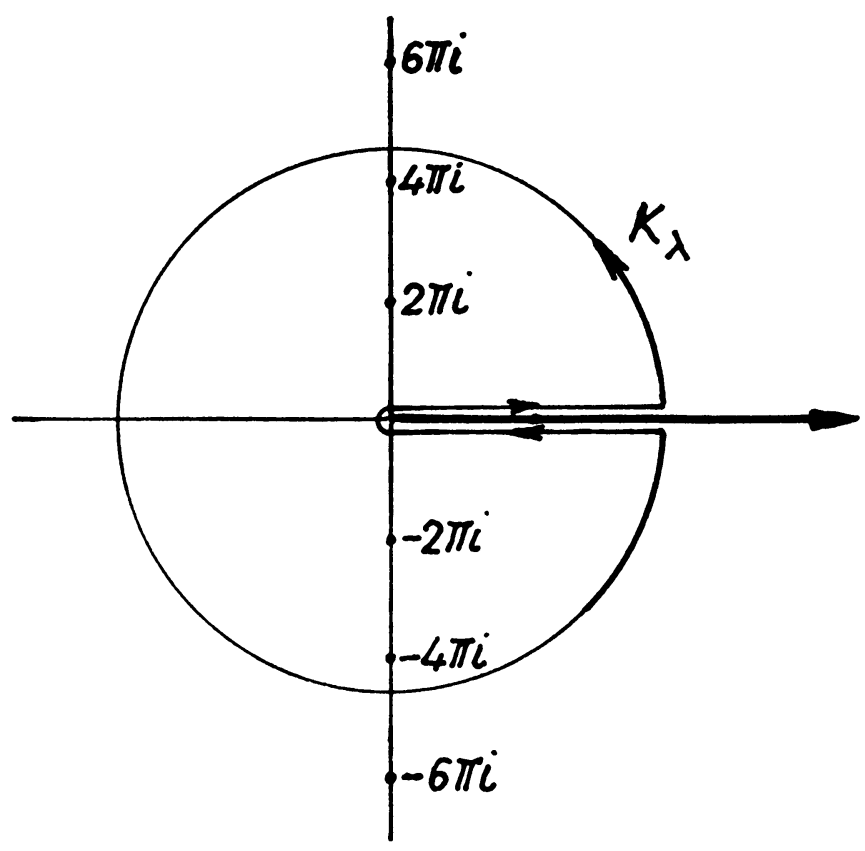

FIGURE 2

In the cut plane, the function $f(z)$ has poles of order 1 at the points $z= \pm 2 n \pi i$ $(n=1,2,3, \cdots)$. If we choose $N$ such that

$$
2 N \pi<\lambda<2(N+1) \pi \quad(N=0,1,2, \cdots)
$$

which is equivalent to $N=[\lambda /(2 \pi)]$, ${ }^{* * *}$ we see that the poles for $n= \pm 1, \pm 2, \cdots$, $\pm N$ if $N \geqq 1$ (or no pole if $N=0$ ) lie inside the contour of Fig. 2, and that the remaining poles, for $n= \pm(N+1), \pm(N+2), \cdots$ lie outside. Applying the residue theorem, and using

$$
\text { Res }\left.f(z)\right|_{z-2 n \pi i}+\left.\operatorname{Res} f(z)\right|_{z--2 n \pi i}=2(2 n \pi)^{*-1} \sin \frac{\pi}{2} s,
$$

we obtain the relation

$$
A(s, \lambda)=\Gamma(1-s)\left\{2^{s} \pi^{s-1} \sin \frac{\pi}{2} s \sum_{n=1}^{N} n^{s-1}-\frac{1}{2 \pi i} \int_{K_{\lambda}} \frac{(-z)^{s-1}}{e^{z}-1} d z\right\}
$$

$* * *[x]$ denotes the largest integer $\leqq x$. 
where the sum over $n$ is zero for $N=0$. For the calculation of the remaining integral over $K_{\lambda}$, we introduce the well-known partial fraction expansion

$$
\frac{1}{e^{z}-1}=\frac{1}{z}-\frac{1}{2}+2 z \sum_{n=1}^{\infty} \frac{1}{z^{2}+(2 n \pi)^{2}}
$$

which converges uniformly in the annulus $2 n \pi<|z|<2(n+1) \pi$ for $n=0,1,2, \cdots$. With $z=\lambda e^{i \theta},(-z)=\lambda e^{i(\theta-x)}, 0 \leqq \theta<2 \pi$, this leads to

$$
\begin{aligned}
& \frac{1}{2 \pi i} \int_{K_{\lambda}} \frac{(-z)^{s-1}}{e^{s}-1} d z \\
& =\frac{\lambda^{*}}{2 \pi}\left\{\frac{\sin \pi s}{s}-\frac{2}{\lambda} \frac{\sin \pi s}{s-1}+2 \lambda \int_{0}^{2 \pi} e^{i(\theta-\pi)(s+1)} \sum_{n=1}^{\infty} \frac{1}{\lambda^{2} e^{2 i \theta}+(2 n \pi)^{2}} d \theta\right\} .
\end{aligned}
$$

Exchanging the sum with the integral and using the substitution $\theta^{\prime}=\theta-\pi$, we find in the case $2 n \pi / \lambda<1$ (i.e., $n=1,2,3, \cdots, N$ if $N \geqq 1$ ) that the integral of the $n$th term in the series is given by

$$
\begin{aligned}
\int_{-\pi}^{\pi} \frac{e^{i(\theta+1) \theta} d \theta}{\lambda^{2} e^{2 i \theta}+(2 n \pi)^{2}} & =\frac{1}{\lambda^{2}} \int_{-\pi}^{\pi} \frac{e^{i(s-1) \theta} d \theta}{1+(2 n \pi / \lambda)^{2} e^{-2 i \theta}} \\
& =\frac{1}{\lambda^{2}} \int_{-\pi}^{\pi} \sum_{i=0}^{\infty}(-1)^{i}\left(\frac{2 n \pi}{\lambda}\right)^{2 i} e^{i(\theta-1-2 i) \theta} d \theta \\
& =-\frac{2}{\lambda^{2}} \sin \pi s \sum_{j=0}^{\infty} \frac{(-1)^{i}}{s-2 j-1}\left(\frac{2 n \pi}{\lambda}\right)^{2 i}
\end{aligned}
$$

and, similarly, for $\lambda /(2 n \pi)<1$ (i.e., $n=N+1, N+2, \cdots)$,

$$
\int_{-\pi}^{\pi} \frac{e^{i(s+1) \theta} d \theta}{\lambda^{2} e^{2 i \theta}+(2 n \pi)^{2}}=-\frac{1}{2 n^{2} \pi^{2}} \sin \pi s \sum_{i=0}^{\infty} \frac{(-1)^{i}}{s+2 j+1}\left(\frac{\lambda}{2 n \pi}\right)^{2 i} \text {. }
$$

Introducing these results into (29) and (27), we have

$A(s, \lambda)=\frac{1}{\pi} \Gamma(1-s)\left\{(2 \pi)^{s} \sin \frac{\pi}{2} s \sum_{n=1}^{N} n^{s-1}\right.$

$$
\begin{array}{r}
-\lambda^{s} \sin \pi s\left[\frac{1}{2 s}-\frac{1}{\lambda(s-1)}-\frac{2}{\lambda} \sum_{n=1}^{N} \sum_{i=0}^{\infty} \frac{(-1)^{j}}{s-2 j-1}\left(\frac{2 n \pi}{\lambda}\right)^{2 i}\right. \\
\left.\left.-2 \lambda \sum_{n=N+1}^{\infty} \frac{1}{(2 n \pi)^{2}} \sum_{i=0}^{\infty} \frac{(-1)^{i}}{s+2 j+1}\left(\frac{\lambda}{2 n \pi}\right)^{2 i}\right]\right\},
\end{array}
$$

where again the sums from 1 to $N$ are zero for $N=0$. This is Putschbach's formula. The double sums in this formula can be conveniently rearranged, and one finally has $A(s, \lambda)=\frac{1}{\pi} \Gamma(1-s)\left\{(2 \pi)^{s} \sin \frac{\pi}{2} s \sum_{n=1}^{N} n^{-1-1}\right.$

$$
\begin{array}{r}
-\lambda^{\prime} \sin \pi s\left[\frac{1}{2 s}-\frac{1}{\lambda(s-1)}-\frac{2}{\lambda} \sum_{i=0}^{\infty} \alpha_{i}^{(N)} \frac{(-1)^{i}}{s-2 j-1}\left(\frac{2 N \pi}{\lambda}\right)^{2 i}\right. \\
\left.\left.-\frac{\lambda}{2(N+1)^{2} \pi^{2}} \sum_{i=0}^{\infty} \beta_{i}^{(N)} \frac{(-1)^{i}}{s+2 j+1}\left(\frac{\lambda}{2(N+1) \pi}\right)^{2 i}\right]\right\} .
\end{array}
$$


The constants $\alpha_{i}^{(N)}$ and $\beta_{i}^{(N)}$ are defined by

$$
\begin{array}{ll}
\alpha_{i}^{(N)}=\sum_{n=1}^{N}\left(\frac{n}{N}\right)^{2 i} \quad(N \geqq 1), & \alpha_{i}^{(0)}=0 ; \\
\beta_{i}^{(N)}=\sum_{n=N+1}^{\infty}\left(\frac{N+1}{n}\right)^{2 j+2}, & \beta_{i}^{(0)}=\zeta(2 j+2) .
\end{array}
$$

This normalized form has the considerable advantage that

$$
\alpha_{i}^{(N)} \sim 1, \quad \beta_{i}^{(N)} \sim 1
$$

for $j \rightarrow \infty$ (with $N>0$ for $\alpha_{i}^{(N)}$ ). This implies that, for the numerical calculation, one has to store only a limited number of these constants, without necessarily restricting the upper limit of the summation index $j$ to the number of coefficients stored.

A similar procedure can be applied to the function $B(s, \lambda)$, which can be written as

$$
B(s, \lambda)=-\frac{\Gamma(1-s)}{2 \pi i} \int_{C_{B}} \frac{(-z)^{s-1}}{e^{z}+1} d z
$$

Here, the poles of the integrand lie at the points $z= \pm(2 n-1) \pi i(n=1,2,3, \cdots)$. For $(2 N-1) \pi<\lambda<(2 N+1) \pi$ or, equivalently, $N=[(\lambda+\pi) /(2 \pi)]$, we obtain, following the same procedure as for $A(s, \lambda)$, and using the partial fraction expansion

$$
\frac{1}{e^{z}+1}=\frac{1}{2}-2 z \sum_{n=1}^{\infty} \frac{1}{z^{2}+((2 n-1) \pi)^{2}},
$$

the formula

$$
\begin{aligned}
B(s, \lambda)=-\frac{1}{\pi} \Gamma(1-s)\{ & 2 \pi^{2} \sin \frac{\pi}{2} s \sum_{n=1}^{N}(2 n-1)^{s-1} \\
& -\lambda^{s} \sin \pi s\left[\frac{1}{2 s}-\frac{2}{\lambda} \sum_{i=0}^{\infty} \gamma_{i}^{(N)} \frac{(-1)^{i}}{s-2 j-1}\left(\frac{(2 N-1) \pi}{\lambda}\right)^{2 i}\right. \\
& \left.\left.-\frac{2 \lambda}{(2 N+1)^{2} \pi^{2}} \sum_{i=0}^{\infty} \delta_{i}^{(N)} \frac{(-1)^{i}}{s+2 j+1}\left(\frac{\lambda}{(2 N+1) \pi}\right)^{2 i}\right]\right\} .
\end{aligned}
$$

Here, the constants $\gamma_{i}^{(N)}$ and $\delta_{i}^{(N)}$ are defined by

$$
\begin{aligned}
& \gamma_{i}^{(N)}=\sum_{n=1}^{N}\left(\frac{2 n-1}{2 N-1}\right)^{2 i} \quad(N \geqq 1), \quad \gamma_{i}^{(0)}=0 ; \\
& \delta_{i}^{(N)}=\sum_{n=N+1}^{\infty}\left(\frac{2 N+1}{2 n-1}\right)^{2 i+2}, \quad \delta_{i}^{(0)}=\left(1-2^{-2 i-2}\right) \zeta(2 j+2) .
\end{aligned}
$$

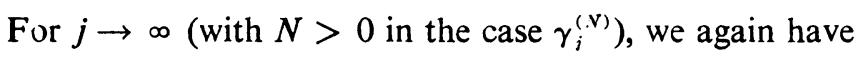

$$
\gamma_{i}^{(N)} \sim 1, \quad \delta_{i}^{(N)} \sim 1 .
$$

The constants $\alpha_{i}^{(N)}, \cdots, \delta_{i}^{(N)}$ can be expressed by Bernoulli numbers, but this does not lead to a significant simplification.

We finally note that $A(s, \lambda)$ and $B(s, \lambda)$ are related by the equation

$$
B(s, \lambda)=A(s, \lambda)-2^{1-\cdot} A(s, 2 \lambda)
$$

which may be obtained from (1) and (4) by using the fact that 


$$
\left(e^{x}+1\right)^{-1}=\left(e^{x}-1\right)^{-1}-2\left(e^{2 x}-1\right)^{-1} .
$$

4. Nontrivial Zeros of $A(s, \lambda)$ and $B(s, \lambda)$. In [1], the first six zero trajectories $\bar{s}_{m}(\lambda)(m=1,2, \cdots, 6)$ were calculated. It was found that only $\bar{s}_{4}(\lambda)$ and $\bar{s}_{0}(\lambda)$ reached zeros of $\zeta(s)$ as $\lambda \rightarrow \infty$, terminating respectively at the first two zeros, $s_{1}=$ $\frac{1}{2}+14.13473 i$ and $s_{2}=\frac{1}{2}+21.02204 i$. A numerical investigation of the trajectories $s_{m}(\lambda)$ for $m=7,8, \cdots, 14$ has given the result shown in Fig. 3. Since the behaviour of the trajectories is fairly regular for $\sigma \lesssim-5$, only the region around $\sigma=0$ has been investigated and only the results for this region are plotted in Fig. 3. One sees that, in addition to $\bar{s}_{4}(\lambda)$ and $\bar{s}_{6}(\lambda)$, the trajectories $\bar{s}_{8}(\lambda), \bar{s}_{10}(\lambda), \bar{s}_{12}(\lambda)$, and $\bar{s}_{14}(\lambda)$ reach the zeros $s_{3}=\frac{1}{2}+25.01086 i, s_{4}=\frac{1}{2}+30.42488 i, s_{5}=\frac{1}{2}+32.93506 i$, and $s_{6}=\frac{1}{2}+37.58618 i$, respectively. The trajectories $\bar{s}_{7}(\lambda), \bar{s}_{9}(\lambda), \bar{s}_{11}(\lambda)$, and $\bar{s}_{13}(\lambda)$ approach the zero trajectories $\tilde{s}_{5}(\lambda), \tilde{s}_{6}(\lambda), \tilde{s}_{7}(\lambda)$, and $\tilde{s}_{8}(\lambda)$ of the incomplete gamma function $P(s, \lambda)$, respectively. The fact that for $4 \leqq m \leqq 14$ all the even-numbered trajectories reach a zero $s_{M}$ of $\zeta(s)$, whereas all the odd-numbered trajectories approach a zero trajectory of $P(s, \lambda)$, is somewhat surprising for its regularity. It is, however, by no means certain that this will be a general law. Consequently, a new question arises-namely how far this behaviour will continue. $\dagger$ At present, the investigation of this problem has not been carried further, since the numerical difficulties become too great, as will be explained later.

For the function $B(s, \lambda)$, we note from (3) and (4) that

$$
\left(1-2^{1-s}\right) \zeta(s)=\frac{1}{\Gamma(s)} \lim _{\lambda \rightarrow \infty} \int_{0}^{\lambda} \frac{x^{s-1}}{e^{x}+1} d x .
$$

Since the factor $\phi(s)=1-2^{1-s}$ on the left-hand side of this equation vanishes in the upper half-plane $t>0$ for

$$
s_{k}^{0}=1+\frac{2 \pi k}{\log 2} i=1+9.06472 k i \quad(k=1,2, \cdots)
$$

and since we have $\left|\zeta\left(s_{k}^{0}\right)\right|<\infty$ and $0<\left|\Gamma\left(s_{k}^{0}\right)\right|<\infty$, it follows that

$$
\int_{0}^{\infty} \frac{x^{8 x^{0}-1}}{e^{x}+1} d x=\int_{0}^{\infty} \frac{x^{(2 \pi k / \log 2) i}}{e^{x}+1} d x=0 \quad(k=1,2, \cdots)
$$

Therefore, one has to expect that some of the trajectories $\hat{s}_{m}(\lambda)$ defined by $B\left(\hat{s}_{m}(\lambda), \lambda\right)$ $=0$ may end at the points $s_{k}^{0}$. As will be seen, this is indeed the case.

The problem of calculating the trajectories $\hat{s}_{m}(\lambda)$ is treated in a similar way to that of $\bar{s}_{m}(\lambda)$ [1]. From preliminary calculations, which were carried out very conveniently on the CERN interactive GAMMA system, using the formula

$$
B(s, \lambda) \approx \frac{\lambda^{s}}{\Gamma(s)}\left\{\frac{1}{2 s}+\sum_{n=1}^{30} \frac{1-2^{2 n}}{(2 n) !} B_{2 n} \frac{\lambda^{2 n-1}}{s+2 n-1}\right\}
$$

for real negative $s$, it was found that, just as $A(s, \lambda)$ oscillates about $\zeta(s)$, so $B(s, \lambda)$ oscillates about $\zeta^{*}(s)$ defined in (5). Both functions have the same values at $s=-k$

† For instance, it would be interesting to know whether there is any special behaviour of the trajectories near a zero-free Gram interval of $\zeta(s)$ (see, e.g., Lehmer [11] or Barkley Rosser et al. [12]). The first of these intervals is around $t \approx 282$ and therefore far beyond the range of the present calculations. 
Zero trajectories of
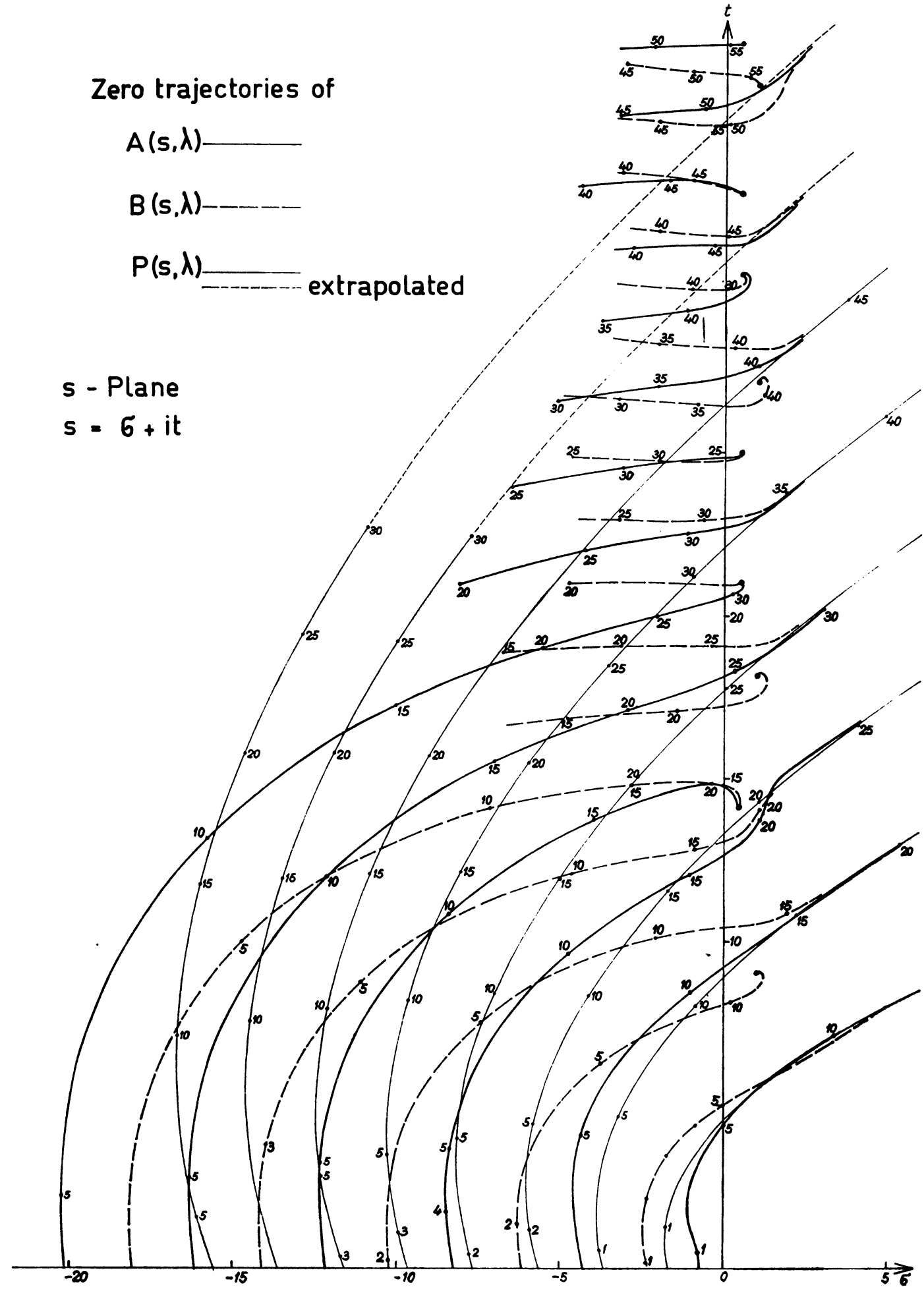

FIGURE 3 
$(k=0,1,2, \cdots)$. One finds also that the starting point $\hat{s}_{1}^{*}$ of $\hat{s}_{1}(\lambda)$ lies around $s \approx-2.35$, for $\lambda_{1}^{*} \approx 0.986$. A further investigation with the GAMMA system gave approximations for the starting values of the higher order trajectories. A detailed numerical calculation then provided accurate starting points $\hat{s}_{m}^{*}$ for the trajectories $\hat{s}_{m}(\lambda)$ for $m=1,3, \cdots, 5$ as given in Table 1 .

TABLE 1

\begin{tabular}{lcc}
\hline$m$ & $\hat{\lambda}_{m}^{*}$ & $\hat{s}_{m}^{*}=\hat{s}_{m}\left(\hat{\lambda}_{m}^{*}\right)$ \\
\hline 1 & 0.98656 & -2.34145 \\
2 & 1.66221 & -6.20124 \\
3 & 1.98928 & -10.14943 \\
4 & 2.18907 & -14.12026 \\
5 & 2.32552 & -18.10126 \\
\hline
\end{tabular}

It seems that $\hat{s}_{m}^{*}=2-4 m-\epsilon(m)$ for $m \rightarrow \infty$, where $\epsilon(m)=O(1)$. No attempt was made to prove this relation.

A systematic numerical investigation of the first 17 zero trajectories $\hat{s}_{m}(\lambda)$ gives the result shown in Fig. 3. There are, in fact, three classes of curves, namely those which end in a zero $s_{M}$ of $\zeta(s)$ on the line $\sigma=\frac{1}{2}$, those which approach a zero trajectory of $P(s, \lambda)$, and those which end in a zero $s_{k}^{0}$ of $\phi(s)=1-2^{1-s}$ on the line $\sigma=1$. The following table marks with an asterisk the behaviour of $\hat{s}_{m}(\lambda)$ as $\lambda \rightarrow \infty$.

TABLE 2

\begin{tabular}{lllllllllllllllllcccc}
\hline$m$ & 1 & 2 & 3 & 4 & 5 & 6 & 7 & 8 & 9 & 10 & 11 & 12 & 13 & 14 & 15 & 16 & 17 \\
\hline $\begin{array}{l}\zeta(s) \\
P(s, \lambda)\end{array}$ & $*$ & & $*$ & $*$ & & & $*$ & & $*$ & & & $*$ & & $*$ & & $*$ & \\
$\phi(s)$ & & $*$ & & & & $*$ & & & & & $*$ & & & & & & $*$ \\
\hline
\end{tabular}

It is, of course, impossible, to deduce a general behaviour for higher $m$ from the calculated results. It might be that such a behaviour does not exist in the case of $B(s, \lambda)$, since we have here the additional zeros $s_{k}^{0}$, which are equally spaced and which interfere with the unequally spaced zeros of $\zeta(s)$.

5. The Numerical Calculation of the Zeros of $A(s, \lambda)$ and $B(s, \lambda)$. As was already stated, the numerical evaluation of the integrals in formulae (10) of reference [1] and (16) becomes very time-consuming for increasing values of $\lambda$ and $t$. The results for $A(s, \lambda)$ and $B(s, \lambda)$ can be obtained in a much faster way by using the expressions (33) and (37), provided that $\lambda$ is not too near to an even or odd multiple of $\pi$, respectively. These representations, however, have the disadvantage that they are very sensitive to the cancellation of terms. In particular, the quantities in the 
TABLE 3

\begin{tabular}{cccc}
\hline$J_{\alpha}$ & $J_{\beta}$ & $J_{\gamma}$ & $J_{\delta}$ \\
\hline $32 N-18$ & $32 N+46$ & $32 N-34$ & $32 N+30$ \\
$(N \geqq 2)$ & $(N \geqq 0)$ & $(N \geqq 2)$ & $(N \geqq 0)$ \\
\hline
\end{tabular}

square brackets are likely to cancel to a large extent, especially for increasing $t$. Since these brackets are multiplied by large quantities and the results are added to others, the cancellation becomes quite dangerous. All the computations were therefore performed in double-precision arithmetic on a CDC 6600 computer, corresponding to about 28 decimal digits. The constants $\alpha_{i}^{(N)}, \cdots, \delta_{i}^{(N)}$ were computed beforehand and stored in a data file. Since they approach unity for $j \rightarrow \infty$, only restricted numbers $J_{\alpha}, \cdots, J_{\delta}$ of them have to be stored. As an indication, the numbers $J_{\alpha}, \cdots, J_{\delta}$, required for 28-digit precision, are given as functions of $N(N \leqq 10)$ in Table 3 .

TABLE 4

Zero Trajectories for the Incomplete Zeta Function $B(s, \lambda)$

\begin{tabular}{|c|c|c|c|c|c|}
\hline \multicolumn{3}{|c|}{$\hat{s}_{1}(\lambda)$} & \multicolumn{3}{|c|}{$\hat{s}_{2}(\lambda)$} \\
\hline$\lambda$ & $\sigma$ & $t$ & $\lambda$ & $\sigma$ & $t$ \\
\hline 1 & -2.34631 & 0.16772 & & & \\
\hline 2 & -2.36578 & 2.12190 & 2 & -6.29432 & 1.33751 \\
\hline 3 & -1.72637 & 3.45312 & 3 & -5.87078 & 3.65737 \\
\hline 4 & -0.86760 & 4.35556 & 4 & -4.87320 & 5.24436 \\
\hline 5 & -0.01500 & 4.97944 & 5 & -3.77683 & 6.27204 \\
\hline 6 & 0.77159 & 5.45541 & 6 & -2.75156 & 6.94395 \\
\hline 7 & 1.49273 & 5.86507 & 7 & -1.84050 & 7.39900 \\
\hline 8 & 2.16699 & 6.25539 & 8 & -1.04691 & 7.72099 \\
\hline 9 & 2.81776 & 6.65159 & 9 & -0.36702 & 7.96112 \\
\hline 10 & 3.46874 & 7.06278 & 10 & 0.19963 & 8.15598 \\
\hline 11 & 4.13880 & 7.48443 & 11 & 0.65044 & 8.33890 \\
\hline 12 & 4.83593 & 7.90432 & 12 & 0.98185 & 8.54174 \\
\hline 13 & 5.55766 & 8.31209 & 13 & 1.17830 & 8.78700 \\
\hline 14 & 6.29755 & 8.70355 & 14 & 1.17655 & 9.04118 \\
\hline 15 & 7.05021 & 9.07888 & 15 & 1.04917 & 9.10687 \\
\hline 16 & 7.81230 & 9.43983 & 16 & 1.00583 & 9.08637 \\
\hline 17 & 8.58191 & 9.78824 & 17 & 0.99793 & 9.07256 \\
\hline 18 & 9.35796 & 10.12569 & 18 & 0.99797 & 9.06691 \\
\hline 19 & 10.13977 & 10.45343 & 19 & 0.99896 & 9.06511 \\
\hline 20 & 10.92690 & 10.77242 & 20 & 0.99959 & 9.06469 \\
\hline
\end{tabular}


TABLE 4 (continued)

\begin{tabular}{|c|c|c|c|c|c|}
\hline \multicolumn{3}{|c|}{$\hat{s}_{3}(\lambda)$} & \multicolumn{3}{|c|}{$\hat{s}_{4}(\lambda)$} \\
\hline$\lambda$ & $\sigma$ & $t$ & $\lambda$ & $\sigma$ & $t$ \\
\hline 2 & -10.15367 & 0.22596 & & & \\
\hline 3 & -9.93496 & 3.74940 & 3 & -13.97332 & 3.79136 \\
\hline 4 & -8.79853 & 6.04542 & 4 & -12.71326 & 6.79974 \\
\hline 5 & -7.43585 & 7.53915 & 5 & -11.09646 & 8.76752 \\
\hline 6 & -6.12305 & 8.50652 & 6 & -9.50870 & 10.04044 \\
\hline 7 & -4.93709 & 9.14708 & 7 & -8.06649 & 10.87712 \\
\hline 8 & -3.88350 & 9.58480 & 8 & -6.78683 & 11.44142 \\
\hline 9 & -2.94561 & 9.89391 & 9 & -5.65500 & 11.83336 \\
\hline 10 & -2.10115 & 10.11850 & 10 & -4.64905 & 12.11424 \\
\hline 11 & -1.32595 & 10.28424 & 11 & -3.74811 & 12.32250 \\
\hline 12 & -0.59121 & 10.40494 & 12 & -2.93489 & 12.48324 \\
\hline 13 & 0.14854 & 10.48893 & 13 & -2.19629 & 12.61402 \\
\hline 14 & 0.98641 & 10.58553 & 14 & -1.52369 & 12.72861 \\
\hline 15 & 1.87653 & 10.89696 & 15 & -0.91313 & 12.83986 \\
\hline 16 & 2.63291 & 11.31852 & 16 & -0.36519 & 12.96199 \\
\hline 17 & 3.32475 & 11.75021 & 17 & 0.11621 & 13.11227 \\
\hline 18 & 3.99550 & 12.18137 & 18 & 0.52571 & 13.31233 \\
\hline 19 & 4.66219 & 12.60831 & 19 & 0.85593 & 13.59359 \\
\hline 20 & 5.33115 & 13.02867 & 20 & 1.09183 & 14.04000 \\
\hline 21 & 6.00460 & 13.44152 & 21 & 1.59381 & 14.81908 \\
\hline 22 & 6.68342 & 13.84677 & 22 & 2.27519 & 15.31490 \\
\hline 23 & 7.36803 & 14.24460 & 23 & 2.89424 & 15.75818 \\
\hline 24 & 8.05865 & 14.63517 & 24 & 3.49212 & 16.20113 \\
\hline 25 & 8.75523 & 15.01862 & 25 & 4.09019 & 16.65080 \\
\hline
\end{tabular}

It is essential that these constants are calculated as accurately as possible. The $\alpha_{i}^{(N)}$ and $\gamma_{i}^{(N)}$ are finite sums, and no particular problem arises. For $\beta_{i}^{(N)}$ and $\delta_{i}^{(N)}$, however, either infinite sums have to be evaluated or the relations

$$
\beta_{i}^{(N)}=(N+1)^{2 i+2}\left[\zeta(2 j+2)-\sum_{n=1}^{N} \frac{1}{n^{2 j+2}}\right],
$$

$$
\delta_{i}^{(N)}=(2 N+1)^{2 i+2}\left[\left(1-2^{-2 i-2}\right) \zeta(2 j+2)-\sum_{n=1}^{N} \frac{1}{(2 n-1)^{2 j+2}}\right]
$$

have to be used. For $j \geqq 3$, approximations to the infinite sums were calculated directly, since it is clear that the square brackets in the above formulas are a source of cancellation errors. For $j=0,1,2$, however, the convergence of the series (34) and (38) is too slow. For these cases, the help of Mr. Wim Klein (CERN) is acknowledged. He calculated the constants $\beta_{i}^{(N)}$ and $\delta_{i}^{(N)}$ for $j=0,1,2$ and $N=1(1) 10$ rapidly and accurately with paper and pencil from the Eqs. (45) and (46), carrying 
TABLE 4 (continued)

\begin{tabular}{|c|c|c|}
\hline \multicolumn{3}{|c|}{$\hat{s}_{5}(\lambda)$} \\
\hline$\lambda$ & $\sigma$ & $t$ \\
\hline 3 & -17.99851 & 3.80230 \\
\hline 4 & -16.62308 & 7.52454 \\
\hline 5 & -14.75815 & 9.96953 \\
\hline 6 & -12.89947 & 11.55151 \\
\hline 7 & -11.20337 & 12.58729 \\
\hline 8 & -9.69808 & 13.27988 \\
\hline 9 & -8.36947 & 13.75403 \\
\hline 10 & -7.19261 & 14.08642 \\
\hline 11 & -6.14263 & 14.32491 \\
\hline 12 & -5.19793 & 14.49989 \\
\hline 13 & -4.34067 & 14.63099 \\
\hline 14 & -3.55632 & 14.73087 \\
\hline 15 & -2.83307 & 14.80738 \\
\hline 16 & -2.16095 & 14.86457 \\
\hline 17 & -1.53093 & 14.90257 \\
\hline 18 & -0.93321 & 14.91559 \\
\hline 19 & -0.35389 & 14.88426 \\
\hline 20 & 0.23114 & 14.73109 \\
\hline 21 & 0.47252 & 14.28953 \\
\hline 22 & 0.47046 & 14.17194 \\
\hline 23 & 0.48427 & 14.14051 \\
\hline 24 & 0.49402 & 14.13347 \\
\hline 25 & 0.49838 & 14.13319 \\
\hline
\end{tabular}

35 decimals and taking as basic numbers for $\zeta(2 j+2)$ the 35-decimal values of $\pi^{2}$ and $\pi^{4}$ as given in the tables of Fletcher et al. [13].

The details of the calculation procedure for the zero trajectories are already described in [1]. In order to make programming of the expressions for $A(s, \lambda)$ and $B(s, \lambda)$ easier and to avoid splitting into real and imaginary parts, a package of "double-precision complex" subroutines has been written and used in the programs.

For the trajectories which lie in the region $t \gtrsim 35$, the cancellation problem becomes more and more serious for the expressions (33) and (37), in particular for $B(s, \lambda)$. In this region, the formulae (10) of reference [1] and (16) have been used for the calculation or for checking purposes. The numerical integrations were carried out by a program based on the modified Clenshaw-Curtis algorithm developed by Håvie [14], [15]. It was found in this connection, that the substitution $\xi=\log x$ in the integrals reduces the calculation time considerably. It was, however, not possible to use these expressions for further calculation in the region above $t \gtrsim 38$, since practically all the significant digits cancel between the sum and the integral.

Table 4 gives five-digit values of the zeros of $B(s, \lambda)$ for the first five trajectories $\hat{s}_{m}(\lambda)$. 


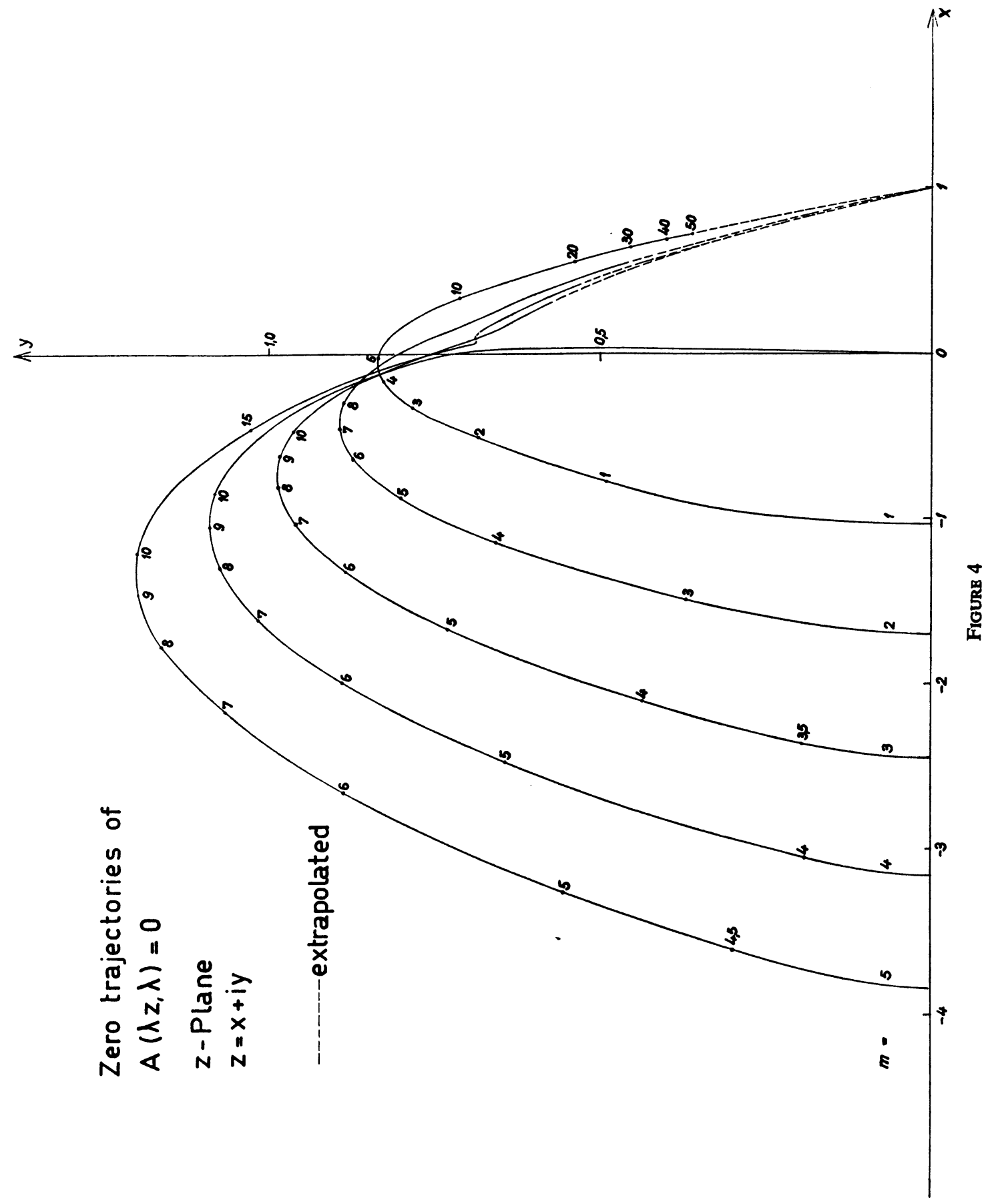


6. A Plot of the Zeros of $A(\lambda z, \lambda)$. It has been seen that some of the zero trajectories of $A(s, \lambda)$ and $B(s, \lambda)$ approach a zero trajectory of $P(s, \lambda)$. In a littleknown paper, Mahler [16] investigated theoretically the behaviour of the zero trajectories $z_{m}(\lambda)=\tilde{s}_{m}(\lambda) / \lambda$ of $P(\lambda z, \lambda)$ in the $z$-plane. In particular, he found that the moduli of the starting points $\tilde{z}_{m}^{*}=\tilde{S}_{m}^{*} / \tilde{\lambda}_{m}^{*}$ (see Table 2 in [1]) on the real negative axis decrease with increasing $m$, approaching a limiting value; and that the curves $z_{m}(\lambda)$ cluster towards a limiting curve for $m \rightarrow \infty$. In addition, he showed that the $z_{m}(\lambda)$ end in the point $z=1$ for $\lambda \rightarrow \infty$ and all $m . \dagger \dagger$

In Fig. 4, the first five zero trajectories $\bar{z}_{m}(\lambda)=\bar{s}_{m}(\lambda) / \lambda$ of $A(\lambda z, \lambda)$ are given. The behaviour of these curves is different from those of $P(\lambda z, \lambda)$ (apart from the irregularities due to the zeros of $\zeta(s)$ ) insofar as the moduli of the starting points $\bar{z}_{m}^{*}=\bar{S}_{m}^{*} / \bar{\lambda}_{m}^{*}$ (see Table 1 in [1]) on the real negative axis increase with $m$. The zero trajectories of $B(\lambda z, \lambda)$ would give a similar picture.

\section{CERN}

Geneva, Switzerland

1. K. S. Kölbig, "Complex zeros of an incomplete Riemann zeta function and of the incomplete gamma function," Math. Comp., v. 24, 1970, pp. 679-696.

2. Y. L. Luke, The Special Flinctions and their Approximations. Vol. II, Math. in Sci. and Engineering, vol. 53, Academic Press, New York, 1969. MR 40 \#2909.

3. E. W. NG, C. J. Devine \& R. F. TOOPER, "Chebyshev polynomial expansion of BoseEinstein functions of order 1 to 10," Math. Comp., v. 23, 1969, pp. 639-644.

4. E. W. NG \& C. J. DEvine, "On the computation of Debye functions of integer orders," Math. Comp., v. 24, 1970, pp. 405-407.

5. R. B. Dingle, "The Fermi-Dirac integrals," Appl. Sci. Res. B, v. 6, 1957, pp. 225239. MR 19, 133.

6. R. B. Dingle, "The Bose-Einstein integrals," Appl. Sci. Res. B, v. 6, 1957, pp. 240-244. MR 19, 133.

7. W. J. CODY \& H. C. THACHeR, JR., "Rational Chebyshev approximations for FermiDirac integrals of order $-1 / 2,1 / 2$ and 3/2," Math. Comp., v. 21, 1967, pp. 30-40.

8. K. S. Köl.big, J. A. Mignaco \& E. Remiddi, "Ön Nielsen's generalized polylogarithms and their numerical calculation," Nordisk Tidskr. Informationsbehandling, v. 10, 1970, pp. 38-74.

9. M. Abramowitz \& I. A. Stegun (Editors), Handbook of Mathematical Functions, with Formulas, Graphs and Mathematical Tables, Nat. Bur. Standards Appl. Math. Series, 55, Superintendent of Documents, U.S. Government Printing Office, Washington, D.C., 1965. MR 31 \# 1400.

10. R. Putschвасн, Untersuchungen über die unvollständige Riemannsche Zetafunktion, Inst. Prakt. Mathematik (IPM), Techn. Hochschule Darmstadt, 1948?. (Unpublished.)

11. D. H. Lehmer, "On the roots of the Riemann zeta-function," Acta Math., v. 95, 1956, pp. 291-298. MR 19, 121; MR 19, 1431.

12. J. Barkley Rosser, L. Schoenfeld \& J. M. Yohe, Rigorous Camputation and the Zeros of the Riemann Zeta-Function, IFIP Congress 68, Edinburgh, Vol. 1, North-Holland, Amsterdam, 1969, pp. 70-76. MR 41 \#2892.

13. A. Fletcher, J. C. P. Miller, L. Rosenhead \& L. J. Comrie, An Index of Mathematical Tables. Vol. 1: Introduction, 2nd ed., Published for Scientific Computing Service, Ltd., London, by Addison-Wesley, Reading, Mass., 1962. MR 26 \#365a.

14. T. HÅvie, "On a modification of the Clenshaw-Curtis quadrature formula," Nordisk Tidskr. Informationsbehandling, v. 9, 1969, pp. 338-350. MR 41 \#1219.

15. T. Hivie, "Further remarks on the modified Clenshaw-Curtis quadrature formula," Nordisk Tidskr. Informationsbehandling. (To appear.)

16. K. Mahler, "Ueber die Nullstellen der unvollstaendigen Gammafunktionen," Rend. Circ. Mat. Palermo, v. 46, 1930, pp. 1-42.

17. K. S. Kölbig, "On the zeros of the incomplete gamma function." (To appear.)

${ }^{\dagger+}$ Details and a plot of some zero trajectories of $P(\lambda z, \lambda)$ will be given elsewhere [17]. 